\title{
The Structure of Locally Finite Two-Connected Graphs
}

\author{
Carl Droms ${ }^{1}$, Brigitte Servatius and Herman Servatius
}

Submitted: May 15, 1995; Accepted: September 4, 1995.

\begin{abstract}
We expand on Tutte's theory of 3-blocks for 2-connected graphs, generalizing it to apply to infinite, locally finite graphs, and giving necessary and sufficient conditions for a labeled tree to be the 3-block tree of a 2-connected graph.
\end{abstract}

Mathematics Subject Classification: 05C40, 05C38, and 05C05.

Key Words and Phrases: Tutte connectivity, 3-block, 3-block tree

\section{Introduction}

Connectivity properties of graphs are among the basic aspects of graph theory. Every graph is the disjoint union of its connected components, and every connected graph is the edge disjoint union of its maximal 2-connected subgraphs, encoded in the block-cutpoint tree. A canonical decomposition for finite 2-connected graphs was given by Tutte [11] in the form of the 3-block tree, and generalized to matroids by Cunningham and Edmonds [1]. Such decompositions are important tools in inductive arguments and constructions. Hopcroft and Tarjan [4] gave an important algorithm for computing the 3-block tree of a graph in $O(V+E)$ time, which is comparable to the complexity of computing other non-canonical decompositions, say the ear decomposition, and is also applicable to matroids. Effective decompositon schemes for graphs of connectivity 3 and higher have been given, but none are canonical, and in Section 6 we argue that none will be forthcoming. The uniqueness of Tutte's construction may be exploited to study the symmetry properties of graphs with low connectivity, [8] and [2], particularly in the case of planar graphs [3]. In this paper we will examine the interpretation of Tutte's decomposition and extend the theory to infinite graphs.

\section{2 -Connectivity}

We are concerned with the structure of locally finite graphs of connectivity less than 4, allowing graphs to have loops and multiple edges. If a graph $G$ has at least 3 vertices and is not a triangle, then $G$ is defined to be $n$-connected if $G$ has girth at least $n$ and any two vertices of $G$ are joined by $n$ internally disjoint paths. To avoid uninteresting special cases, the connectivities of the small graphs in Figure 1 are said to be infinite. This definition of $n$ -

\footnotetext{
${ }^{1}$ Supported by a James Madison University Faculty Summer Research grant.
} 


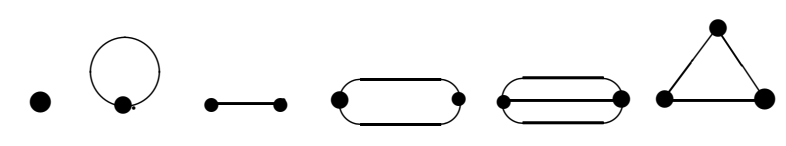

Figure 1: Infinitely connected graphs.

connectivity, also known as Tutte connectivity, was introduced in [9], see also [6], and differs for simple graphs from the usual definition of $n$-connectivity only for $n>3$, with the exception of the special cases in Figure 1, and has the advantage of being generalizable to matroids, [10], and being invariant under dualization.

We call a graph on two vertices connected by parallel edges a multilink. Note, that with the above definition, multilinks on more than three edges are only 2-connected.

For any graph $G$, there is an equivalence relation defined on the edges of $G$ by setting $e \cong e^{\prime}$ if $e$ and $e^{\prime}$ lie on a common cycle, or $e=e^{\prime}$. The equivalence classes are either single edges or induce maximal 2-connected subgraphs of $G$, and are called the blocks (or 2-blocks) of $G$. The block-cutpoint tree is the graph defined on the union of the set of blocks and the set of cutpoints, with a cutpoint adjacent to each of the blocks to which it belongs. It is obvious that this graph is a tree. In general, one might hope that any $k$-connected graph decomposes as the union of subgraphs which are either $k+1$-connected or have at most $k$ vertices. However, this is not the case, since a 2-connected graph may have no non-trivial 3-connected subgraphs at all, as in Figure 2.

In the interest of analogy, therefore, it is preferable to regard a block-cutpoint tree as an encoding of the instructions for assembling a 1-separable graph from simpler pieces using the operation of vertex-union.

Let $A$ and $B$ be graphs and suppose there are functions $f_{A}: e \rightarrow A$ and $f_{B}: e \rightarrow B$, where $e$ is a graph consisting of two vertices 1 and 2 joined by an edge which we will also call $e$. Define the edge amalgam of $A$ and $B$ over $f$-denoted $A+_{f} B$ - to be the graph obtained

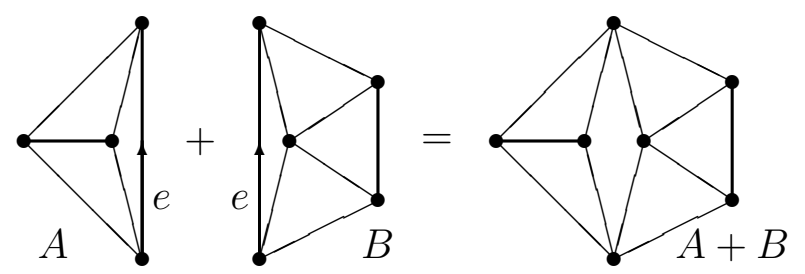

Figure 2: Edge amalgamation.

from the disjoint union of $A$ and $B$ by identifying vertex $f_{A}(1)$ with $f_{B}(1)$, vertex $f_{A}(2)$ with $f_{B}(2)$, and erasing the edges $f_{A}(e)$ and $f_{B}(e)$, see Figure 2. The edge amalgam, also called 2-sum in [6], is analogous to the symmetric difference of sets, and in $A+{ }_{f} B$ we can regard the graph $B-f_{B}(e)$ as taking the place of $f_{A}(e)$ in $A$, and vice versa. Whenever possible, we will indicate the edge functions $f_{A}$ and $f_{B}$ by simply labeling the edges $f_{A}(e)$ and $f_{B}(e)$ the same in $A$ and $B$, in which case we write $A+{ }_{e} B$ or just $A+B$.

It is clear that $A$ and $B$ are 2-connected if and only if $A+{ }_{f} B$ is 2-connected. The edge 
$f_{A}(e)$ is called an amalgamated edge of $A$. If $X$ denotes the subgraph of $A$ obtained by erasing the amalgamated edge, $X$ is also a subgraph of $A+_{f} B$ and we write $A=\bar{X}$.

Edge amalgamation is a commutative operation, so $A+{ }_{f} B \cong B+_{f} A$. but it is not in general associative, since $\left(A+{ }_{f} B\right)+{ }_{g} C$ may be rewritten as $A+_{f}\left(B+_{g} C\right)$ only if $g_{A+B}(e)$ belongs to $B$. If both expressions are defined, they are equal, and note that, since the amalgamated edges are erased, it must be true that $f_{B}(e) \neq g_{B}(e)$. Since the associativity is conditional, it is not in general possible to simply ignore the parentheses in a long expression, in particular whenever some term has more than two amalgamated edges. A more convenient notation for the result of a sequence of edge amalgamations, therefore, is a labeled tree in which the nodes are labeled with graphs, and the edges are labeled with the two functions indicating which edges are amalgamated in the endpoint graphs. Necessarily, the amalgamating edges at any node of this tree must be distinct. We call such a labeled tree $\mathcal{T}$ an edge amalgam tree, and let $G(\mathcal{T})$ denote the graph obtained from the disjoint union of the node labels by amalgamating along the edges determined by the edge functions.

To avoid confusion between $G(\mathcal{T})$ and $\mathcal{T}$, we will hereafter refer to the vertices and edges of an edge amalgam tree as nodes and links, and denote them with Greek as opposed to Roman letters.

For a finite edge amalgam tree $\mathcal{T}$, it is clear that $G(\mathcal{T})$ is 2-connected if and only if the node graphs are 2-connected, and that $G(\mathcal{T})$ is locally finite if and only if the node graphs are locally finite. For infinite trees, however, neither is the case, as can be seen from Figures 3 and 4. Accordingly, we shall give conditions under which an edge amalgam tree defines a locally finite 2-connected graph.

\section{The 3-block tree}

A graph $G$ is said to be a 3-block if it contains at least three edges and is either a circuit, a finite multilink, or a simple, locally finite 3 -connected graph.

Let $\mathcal{T}$ be a countable edge amalgam tree. We call $\mathcal{T}$ a 3 -block tree if the following conditions are satisfied:

1. If $\{\alpha, \beta\} \in E(\mathcal{T})$ then $G_{\alpha}$ and $G_{\beta}$ are not both circuits, nor are they both multilinks.

2. If $\eta=\{\alpha, \beta\}$ is a link in $\mathcal{T}$ amalgamating an edge $e$ in $G_{\alpha}$, then there is a finite subtree $\mathcal{T}^{\prime} \leq \mathcal{T}$ with $\alpha \in \mathcal{T}^{\prime}$ and $\beta \notin \mathcal{T}^{\prime}$, and a path in $G\left(\mathcal{T}^{\prime}\right)$ joining the endpoints of $e$ which is made up entirely of edges unamalgamated in $G(\mathcal{T})$.

3. For each vertex $v$ of $G_{\alpha}, \alpha$ is contained in a finite subtree $\mathcal{T}_{v}<\mathcal{T}$ such that $\operatorname{star}(v)$ in $G\left(\mathcal{T}_{v}\right)$ consists entirely of edges unamalgamated in $\mathcal{T}$.

We impose condition 1 since circuits and multilinks would otherwise have many inequivalent 3-block trees. Condition 2 avoids the situation of Figure 3 in which each amalgamation increases the length of a circuit, resulting in a graph with cut vertices. Condition 3 insures local finiteness, disallowing amalgamations such as in Figure 4. Note that we do not require $\mathcal{T}$ itself to be locally finite; for instance, one may amalgamate a triangle to each of the edges 


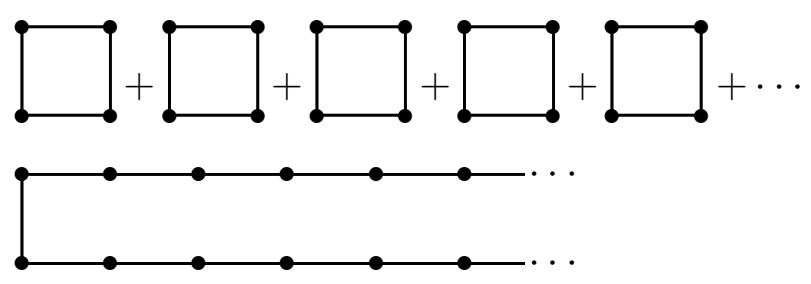

Figure 3:

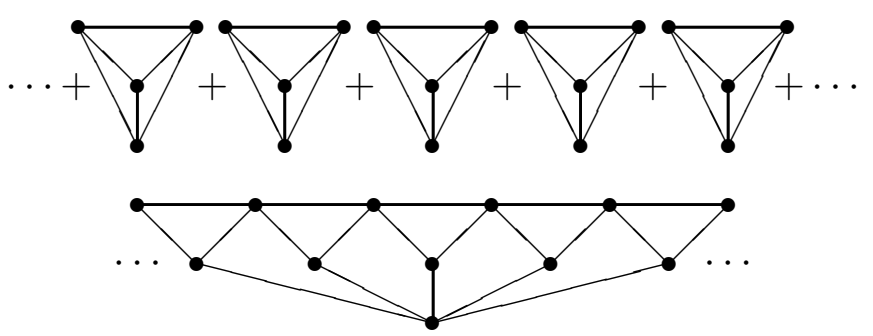

Figure 4:

of an infinite locally finite 3-connected graph (effectively subdividing each edge). In order to describe this graph as $G(\mathcal{T}), \mathcal{T}$ must be an infinite star.

Note also that, while the edges of $G(\mathcal{T})$ are partitioned according to the 3-blocks to which they belong, all edges of a particular 3-block may be amalgamated, in which case the 3-block will not correspond to any collection of edges of $G(\mathcal{T})$.

Lemma 1 If $\mathcal{T}^{\prime}$ is a finite subtree of $\mathcal{T}$, then $G\left(\mathcal{T}^{\prime}\right)$ is homeomorphic to a subgraph of $G(\mathcal{T})$.

Proof: Every edge in $G\left(\mathcal{T}^{\prime}\right)$ which is not in $G(\mathcal{T})$ is amalgamated, so replace each with the path of unamalgamated edges in $G(\mathcal{T})-G\left(\mathcal{T}^{\prime}\right)$ which is guaranteed to exist by condition 2.

Lemma 2 For any 3-block tree $\mathcal{T}, G(\mathcal{T})$ is locally finite and 2-connected.

ProOF: $G(\mathcal{T})$ is locally finite by condition 3 .

Clearly, any finite 3-block tree represents a 2-connected graph. Let $v$ and $w$ be distinct vertices of $G(\mathcal{T})$, and suppose they correspond to vertices $v^{\prime} \in G_{\alpha}$ and $w^{\prime} \in G_{\beta}$. Let $\mathcal{T}^{\prime}$ be any finite subtree of $\mathcal{T}$ containing $\alpha$ and $\beta$. Then $G\left(\mathcal{T}^{\prime}\right)$ is 2-connected, so there are two internally disjoint paths in $G\left(\mathcal{T}^{\prime}\right)$ from $v^{\prime}$ to $w^{\prime}$. Since $G\left(\mathcal{T}^{\prime}\right)$ is homeomorphic to a subgraph of $G(\mathcal{T})$, there are two such paths joining $v$ and $w$ in $G(\mathcal{T})$, as well.

It is our aim to prove

Theorem 1 Any locally finite 2-connected graph $G$ corresponds to a unique 3-block tree $\mathcal{T}$.

We will prove Theorem 1 in two steps. In the next section, we will show how to construct a particular 3-block tree for $G$, and in the one following, we show that this tree is unique. 


\section{Existence of 3-block trees.}

Given a pair of vertices $\{a, b\}$ in a graph $G$, there is an equivalence relation defined on the edges of $G$ by setting $e$ and $e^{\prime}$ equivalent if there is a path in $G$ containing both $e$ and $e^{\prime}$, and which has neither $a$ nor $b$ as an internal vertex. The subgraphs of $G$ which carry the equivalence classes are called the bridges of $G$ with respect to $\{a, b\}$. Two distinct bridges clearly can intersect only at $a$ and $b$, and if $G$ is 2 -connected, their intersection is exactly $\{a, b\}$. $G$ may thus be regarded as the union of its bridges along $\{a, b\}$. However, these bridges may not be themselves 2-connected. If a bridge with respect to $\{a, b\}$ in a 2-connected graph $G$ consists of more than a single edge, then adding a new edge connecting $a$ and $b$ to that bridge does give a 2-connected graph, and we call these graphs the branches of $G$ with respect to $\{a, b\}$.

The deletion of e from $G$, denoted $G-e$, is the graph obtained from $G$ by deleting the edge $e$, but not its endpoints. The contraction of $e$ in $G$, denoted $G \cdot e$, is obtained from $G-e$ by identifying the endpoints of $e$.

Let $G$ be a locally finite 2-connected graph. Choose an arbitrary edge $e$ of $G$, and let $a$ and $b$ be its endpoints. We will construct a 3-block tree $\mathcal{T}_{e}$ with $G\left(\mathcal{T}_{e}\right)=G$.

We call $e$ undeletable if $G-e$ is 1-separable, incontractible if $G \cdot e$ is 1-separable, and ordinary otherwise. An edge $e$ cannot be both undeletable and incontractible. since the first requires that every cycle containing the endpoints of $e$ also contain $e$, while the second insures two vertices $u$ and $v$ so that every path from $u$ to $v$ passes through one of the endpoints of $e$, so neither the two disjoint paths from $u$ to $v$ could contain $e$. Note that if $\mathcal{T}$ is a 3-block tree, then condition 3 , and the fact that edge amalgamation does not destroy cutpoints, guarantees that if the edge $e \in G(\mathcal{T})$ belongs to the 3-block $G_{\alpha}$, then its type -undeletable, incontractible or ordinary - in $G(\mathcal{T})$ is the same as its type in $G_{\alpha}$.

We begin the construction of $\mathcal{T}_{e}$ by defining $\mathcal{T}_{0}$ to consist of a single vertex $\alpha_{0}$ labeled " $G$," with distinguished edge $e$. Then $\mathcal{T}_{0}$ is an edge amalgam tree (though not a 3 -block tree) and $G=G\left(\mathcal{T}_{0}\right)$.

To define $\mathcal{T}_{1}$, we perform what we will call a simple expansion of the vertex $\alpha_{0}$ at the edge $e$; that is, we will replace $\alpha_{0}$ with a star which is an edge-amalgam tree for $G_{v_{0}}$ (though, again, not a 3-block tree.)

First, we define the graph $B_{e}$ - the 3 -block of $G$ containing $e$. This will be the label of the central node of $\mathcal{T}_{1}$. We consider three cases, depending on the type of the edge $e$.

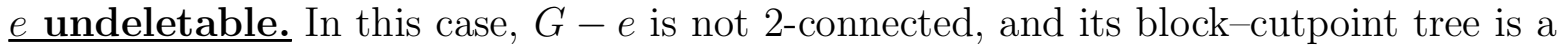
non-trivial path $A_{1}, v_{1}, A_{2}, \ldots, v_{k-1}, A_{k}$, where the vertices $A_{i}$ correspond to blocks of $G-e$ and the $v_{i}$ are the cut vertices of $G-e$. Set $v_{0}=a$ and $v_{k}=b$. Let $B_{e}$ be the $(k+1)$-cycle with vertices $v_{0}, v_{1}, \ldots, v_{k}$, and let $\overline{e_{i}}$ denote the edge of $B_{e}$ joining $v_{i}$ and $v_{i+1}$ (subscripts modulo $n$.) If some $A_{i}$ consists of more than a single edge, we define $\overline{A_{i}}$ to be the graph obtained from $A_{i}$ by adding an edge $\overline{e_{i}}$ joining $v_{i}$ and $v_{i+1}$. $G$ is then obtained by amalgamating each $\overline{A_{i}}$ to $B_{e}$ along $\overline{e_{i}}$, identifying any unamalgamated edges $\overline{e_{i}}$ with the corresponding edge in $G$ (see Figure 5.)

Remark 1. If $A_{i}$ is not a single edge then it is 2-connected (since it is a block of $G-e$ ), so the edge $\overline{e_{i}}$ is not undeletable in $\overline{A_{i}}$. 


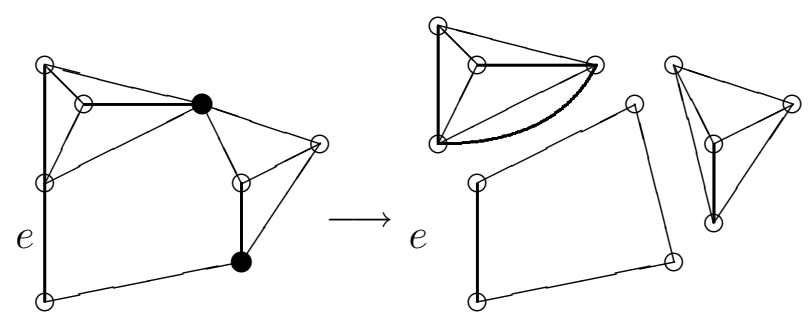

Figure 5: Simple expansion at an undeletable edge.

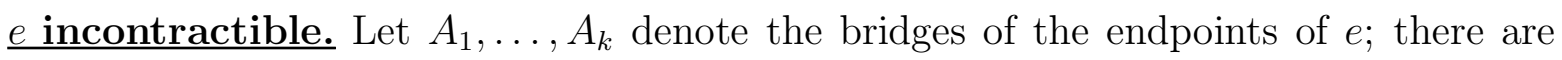
only finitely many of them, since $G$ is locally finite. Then $k \geq 3$ and one of the $A_{i}$ is the edge $e$ itself. If $A_{i}$ consists of more than a single edge, let $\overline{A_{i}}$ denote the graph $A_{i}$ with a new edge $\overline{e_{i}}$ joining $a$ and $b$. If $A_{i}$ is a single edge, set $\overline{e_{i}}=A_{i}$. Let $B_{e}$ denote the multilink consisting of $e$ together with all the edges " $\bar{e}_{i}$ " $G$ is again the amalgam of $B_{e}$ with the graphs $\overline{A_{i}}$ along the edges $\overline{e_{i}}$ (see Figure 6. )

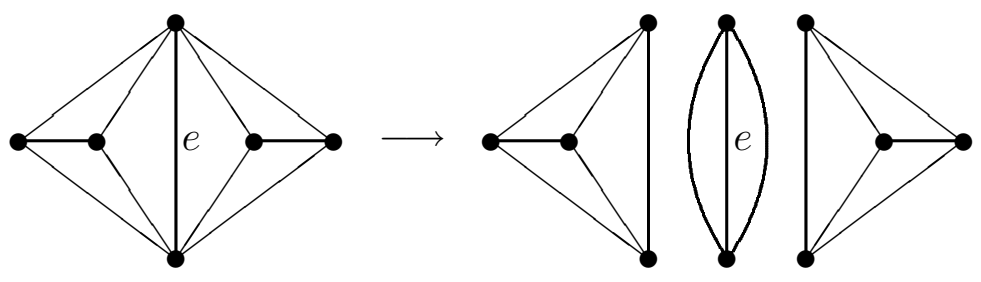

Figure 6: Simple expansion at an incontractible edge.

Remark 2. Since $A_{i}$ has a single bridge with respect to $\{a, b\}, \overline{e_{i}}$ is not incontractible in $\overline{A_{i}}$.

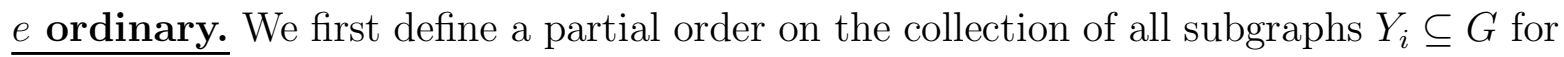
$\overline{\text { which } G=\overline{X_{i}}}+\overline{Y_{i}}$ for some $X_{i}$ properly containing $e$. Specifically, we set $Y_{j} \preceq Y_{i}$ if $Y_{i}=\bar{Z}+\overline{Y_{j}}$ for some subgraph $Z$. Let $\left\{A_{i}\right\}$ denote the collection of maximal elements with respect to this partial order, and let $a_{i}$ and $b_{i}$ be the vertices of attachment of $A_{i}$.

Lemma 3 Two maximal elements $A_{i}$ and $A_{j}$ intersect in at most one common vertex of attachment.

Proof: Let $G=\overline{X_{i}}+\overline{A_{i}}=\overline{X_{j}}+\overline{A_{j}}$. If $A_{i}$ and $A_{j}$ had both vertices of attachment in common, then we could write $G=\overline{X_{i} \cap X_{j}}+\overline{A_{i} \cup A_{j}}$, and $A_{i} \cup A_{j}=\overline{A_{i}}+\overline{A_{j}}$, contrary to the maximality of $A_{i}$ and $A_{j}$.

Suppose $A_{i}$ and $A_{j}$ have an interior vertex $v$ in common. Then there are two internally disjoint paths joining $v$ and $e$, each passing from $A_{i}$ to $X_{i}$ and also from $A_{j}$ to $X_{j}$. By the above, not both $a_{i}$ and $b_{i}$ can belong to $A_{i}$ nor to $A_{j}$. Choose notation so that the paths are labeled as in Figure 7 . Thus $b_{j} \in A_{i}$ and $b_{i} \in A_{j}$, and every path from any internal vertex of $A_{i}$ to $e$ which does not pass through $a_{i}$ must pass through $b_{i}$, which 


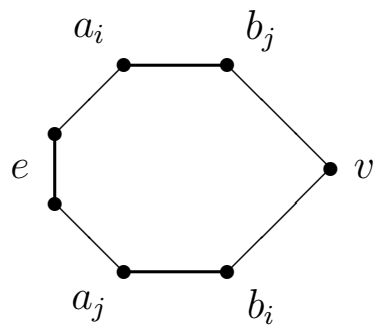

Figure 7:

is in $A_{j}$. Thus, the path must continue through $a_{j}$, since if it continued through $b_{j}$, it would still be within $A_{i}$. But then $\left\{a_{i}, a_{j}\right\}$ separates $A_{i} \cup A_{j}$ from $e$, violating the maximality of $A_{i}$, so $A_{i}$ and $A_{j}$ have no interior vertex in common.

In this case, we form $B_{e}$ by replacing each $A_{i}$ with a new edge $\overline{e_{i}}$ joining $a_{i}$ and $b_{i}$, and we form $\overline{A_{i}}$ by adding the edge $\overline{e_{i}}$ to $A_{i}$. It follows from the maximality of the $A_{i}$ that $B_{e}$ is simple (since otherwise a multilink could be split off of $B_{e}$ ) and that $B_{e}$ is 3 -connected (since if there were a two-cutset, one of its bridges could be split off of $B_{e}$.) Further, $B_{e}$ is locally finite since no vertex of $B_{e}$ has larger valence in $B_{e}$ than it has in $G$. Once again, $G$ is the amalgam of $B_{e}$ with the $\left\{\overline{A_{i}}\right\}$ (see Figure 8.)

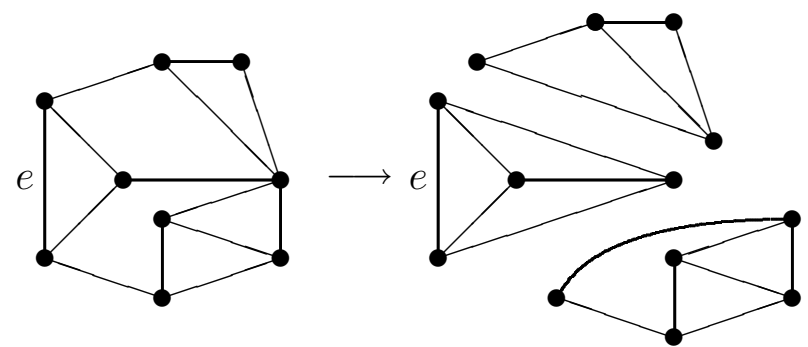

Figure 8: Simple expansion at an ordinary edge.

$\mathcal{T}_{1}$ is a star whose central vertex $\beta$ is labeled " $B_{e}$," and whose pendant vertices $\left\{\alpha_{i}\right\}$ are labeled with the various $\overline{A_{i}}$. Given a link $\eta=\left\{\beta, \alpha_{i}\right\}$ in $\mathcal{T}_{1}$, define the edges $\eta_{\beta}$ and $\eta_{\alpha_{i}}$ to be the edges $\overline{e_{i}}$ in $B_{e}$ and $\overline{A_{i}}$, respectively, with the obvious orientations. Clearly, $G=G\left(\mathcal{T}_{1}\right)$.

Next, suppose $\mathcal{T}_{n}$ has been defined and construct $\mathcal{T}_{n+1}$ by performing, for each pendant node $\alpha$ of $\mathcal{T}_{n}$ which is not a 3-block, a simple expansion of $\alpha$ at the edge $\eta_{\alpha}$ (where $\eta$ is the unique edge of $\mathcal{T}_{n}$ incident to $\alpha$ ).

Then $G=G\left(\mathcal{T}_{n+1}\right)$, and each nonpendant node of $\mathcal{T}_{n+1}$ is labeled with a 3-block. Moreover, by remarks 1 and 2 above, no two adjacent nodes of $\mathcal{T}_{n+1}$ are labeled with either links or circuits.

Let $\mathcal{T}_{e}=\lim \mathcal{T}_{n}$

Let $\mathcal{T}_{n}^{0}$ denote the edge amalgam tree obtained from $\mathcal{T}_{n}$ by removing the pendant nodes which are not 3-blocks and let $G^{0}\left(\mathcal{T}_{n}\right)$ be the graph obtained from $G\left(\mathcal{T}_{n}^{0}\right)$ by removing the edge $\eta_{\alpha}$ for each pendant link $\eta \in \mathcal{T}_{n}$ with endpoint $\alpha \in \mathcal{T}_{n}^{0}$ (in effect, we remove from $G\left(\mathcal{T}_{n}^{0}\right)$ all those edges which are amalgamated in $G\left(\mathcal{T}_{e}\right)$ ). 
THE ElECtronic JoURnal of COMBinatorics 2 (1995), \#R17

It is clear that the $G^{0}\left(\mathcal{T}_{n}\right)$ constitute an increasing sequence of subgraphs of $G\left(\mathcal{T}_{e}\right)$ and that $\lim G^{0}\left(\mathcal{T}_{n}\right)=G\left(\mathcal{T}_{e}\right)$. It is also clear that the $G^{0}\left(\mathcal{T}_{n}\right)$ are subgraphs of $G$; thus, to prove that $G=G\left(\mathcal{T}_{e}\right)$, it suffices to show

Lemma 4 Each finite subgraph of $G$ is contained in $G^{0}\left(\mathcal{T}_{n}\right)$ for some $n$.

ProOF: It suffices to show that each edge of $G$ belongs to one of the graphs $G_{\alpha}$ for some node $\alpha \in \mathcal{T}$, for then we may choose $n$ so large that $\alpha$ is a node of $\mathcal{T}_{n}^{0}$.

Let $e^{\prime}$ be any edge of $G$. If $e^{\prime} \in B_{e}$, then $e^{\prime} \in G^{0}\left(\mathcal{T}_{1}\right)$, so suppose $e^{\prime} \notin B_{e}$. Let $d$ be the length of a shortest circuit in $G$ containing both $e$ and $e^{\prime}$. Let $\alpha$ be the node of $\mathcal{T}_{1}$ with $e^{\prime} \in G_{\alpha}$, and let $e^{\prime \prime}=\eta_{\alpha}$, where $\eta$ is the link of $\mathcal{T}_{1}$ connecting $\alpha$ to the central vertex. Then the length of a shortest circuit in $G_{\alpha}$ containing both $e^{\prime}$ and $e^{\prime \prime}$ is either $d$ (if $B_{e}$ is a multilink) or strictly less than $d$ (otherwise). Since two multilinks cannot be adjacent in any $\mathcal{T}_{n}$, it follows by induction that $e^{\prime}$ belongs to $G_{\gamma}$ for some node $\gamma \in \mathcal{T}_{n}^{0}$ for some $n$, and since $e^{\prime}$ is not amalgamated, it belongs to $G^{0}\left(\mathcal{T}_{n+1}\right)$.

So we have that $G=G\left(\mathcal{T}_{e}\right)$, and by the lemma, $\mathcal{T}_{e}$ satisfies conditions 2 and 3 , and so $\mathcal{T}_{e}$ is a 3 -block tree representing $G$.

\section{$5 \quad$ Invariance of $\mathcal{T}_{e}$}

Proposition 1 Let $G$ be a locally finite 2-connected graph, and let e be an edge of $G$. If $\mathcal{T}$ is any 3-block tree for $G$, then $\mathcal{T}=\mathcal{T}_{e}$.

ProOF: We will show that the 3-block of $\mathcal{T}$ which contains the edge $e$ is equal to $B_{e}$ in $\mathcal{T}_{e}$. Once this is established, then the subgraphs $A_{i}$ are determined, and an induction shows that, for all $k$, the subtree of $\mathcal{T}$ consisting of all nodes a distance $k$ from the node labeled $B_{e}$ is identical with the corresponding subtree of $\mathcal{T}_{e}$, and the result follows.

Let $\alpha$ be the node of $\mathcal{T}$ whose graph $G_{\alpha}$ contains $e$.

If $e$ is undeletable, then $G_{\alpha}$ is a cycle $C$, and the internal vertices of $C-e$ correspond to cutpoints of $G-e$. Let $e^{\prime}$ be an amalgamated edge along this cycle. Then $e^{\prime}=\eta_{\alpha}$ for some link $\eta$ with endpoint $\alpha$. Let $\beta$ be the other endpoint of $\eta$. Let $\mathcal{T}^{\prime}$ denote the component of $\mathcal{T}-\eta$ containing $\beta$, and let $e^{\prime \prime}=\eta_{\beta}$. Then $e^{\prime \prime}$ is not undeletable in $G\left(\mathcal{T}^{\prime}\right)$, since otherwise, $G_{\beta}$ would be a cycle, as well. Therefore, every cutpoint of $G-e$ is a vertex of $C$, and $G_{\alpha}=C=B_{e}$.

If $e$ is incontractible, then $G_{\alpha}$ is a multilink, and each component of $\mathcal{T}-\alpha$ corresponds to a union of some of the bridges of the endpoints of $e$. If some such component, say $\mathcal{T}^{\prime}$, corresponds to more than one branch, then its amalgamated edge $e^{\prime}$ will be incontractible in $G\left(\mathcal{T}^{\prime}\right)$, and so the 3 -block $G_{\beta}$ containing it is a multilink. But $\beta$ is adjacent to $\alpha$ in $\mathcal{T}$, so this is impossible. Therefore, each component of $\mathcal{T}-\alpha$ corresponds to exactly one branch, and so $G_{\alpha}=B_{e}$.

If $G_{\alpha}$ is simple, locally finite and 3-connected, then $e$ is ordinary in $G$. Thus, $B_{e}$ is also simple, locally finite and 3-connected. We must show that $B_{e}=G_{\alpha}$.

Let $\eta$ be a link in $\mathcal{T}$ joining $\alpha$ with, say, $\beta$, and let $\mathcal{T}^{\prime}$ be the component of $\mathcal{T}-\alpha$ containing $\beta$. Then it is clear that the subgraph $G\left(\mathcal{T}^{\prime}\right)-\eta_{\beta}$ of $G(\mathcal{T})$ is maximal with respect to the partial order defined earlier, and the same is true for every link of $\mathcal{T}$ incident with $\alpha$. Since these 
maximal elements are uniquely determined once $e$ has been chosen, it follows that $G_{\alpha}=B_{e}$.

\section{Higher connectivity}

The decomposition of a 2-connected graph into its 3-block tree is distinguished from other decompositions of 2-connected graphs by the fact that the 3-block tree is uniquely defined. Thus any symmetry exhibited by the graph $\mathcal{T}(G)$ will be reflected in the tree $\mathcal{T}$. Specifically, any automorphism of $G(\mathcal{T})$ induces an automorphism of $\mathcal{T}$. This is also true in the case of the block-cutpoint tree, however, analogous decompositions of graphs of higher connectivity, see [7], are not unique.

Theorem 2 Let $G$ be a simple 1-connected vertex transitive graph. Then either $G$ is 2connected or its block-cutpoint tree is infinite.

ProOF: Let $\mathcal{T}$ be the block-cutpoint tree. If $\mathcal{T}$ is finite, then it has a center, which is an edge or a vertex. The center cannot be an edge, since the cutpoint corresponding to one of its endpoints would be distinguished in $G$. Similarly, the center cannot be a node corresponding to a cutpoint, so the center is a node corresponding to a block. Moreover, the central block must contain every vertex of $G$, so any other block can at most be a loop, and since $G$ is simple, there is only one block.

A similar result is also true for 2-connected graphs.

Theorem 3 Let $G$ be a simple 2-connected vertex transitive graph. Then either $G$ is either a cycle, 3-connected or its 3-block tree is infinite.

ProOF: Let $\mathcal{T}$ be the 3-block tree. If $\mathcal{T}$ is finite, then it has a center, which is an edge or a vertex. The center cannot be a link of $\mathcal{T}$, since the two endpoints of the amalgamated edge corresponding to it would be distinguished, and $G$ is not a multilink.

Thus the center is a node, and that node cannot represent a multilink, since, again, its endpoints would be distinguished in $G$. Every vertex of $G$ must be in the 3-block of the central node, hence every other 3 -block is a mulitlink. Since $G$ is simple, $\mathcal{T}$ consists of exactly one 3 -block, hence $G$ is a cycle or 3-connected.

The pattern of these two proofs indicates the "meta-result" that there cannot be a canonical tree decomposition of 3-connected graphs in analogy with two and one connectivity, since we know there exists infinitely many finite 3-connected vertex transitive graphs which are not 4-connected, e.g. the Cayley graphs of finite groups with three generators, and these graphs would have to be indecomposable.

\section{Authors' addresses:}

James Madison University, Harrisonburg, VA 22807, cdroms@cayuga.math.jmu.edu Worcester Polytechnic Institute, Worcester, MA 01609-2280, bservat@math.wpi.edu 11 Hackfeld Rd, Worcester MA 01609, jhs@cayuga.math.jmu.edu 
THE EleCtronic Journal of COMBinatorics 2 (1995), \#R17

\section{References}

[1] W. H. Cunningham and J. Edmonds, A combinatorial decomposition theory, Canad. J. Math. 32, 1980, 734-765.

[2] C. Droms. B. Servatius and H. Servatius, Connectivity of Groups, preprint

[3] C. Droms. B. Servatius and H. Servatius, Planar Cayley Graphs, preprint

[4] J. E. Hopcroft and R. E. Tarjan, Dividing a graph into triconnected components, SIAM J. Comput. 3, 2, 135-158, 1973.

[5] D. J. Kleitman, Methods of investigating the connectivity of large graphs, IEEE Trans. Circuit Theory, 16, 232-233, 1969.

[6] J. G. Oxley, Matroid Theory, Oxford Univ. Press (1992).

[7] J. G. Oxley and H. Wu, On the structure of 3-connected matroids and graphs, Preprint, 1995.

[8] B. Servatius and H. Servatius, Self-dual graphs, to appear in Discrete Math.

[9] W. T. Tutte, Connectivity in Graphs, University of Toronto Press (1966).

[10] W. T. Tutte, Connectivity in Matroids, Canad. J. Math., 18, 1966, 1301-1324.

[11] W. T. Tutte, Graph Theory, Encyclopedia of Mathematics, Vol. 21, Cambridge University Press, Cambridge (1984).

[12] H. Whitney, 2-isomorphic graphs, Amer. J. Math. 55, 1933, 245-254. 